\title{
A belief-based approach to spectrum occupancy mapping
}

Tricia J Willink

\begin{abstract}
Maintaining a map of signal power levels is one approach to dynamic spectrum management, but generating it in a complex environment with unknown emitters using sensor measurements is challenging. Precise representation of signal levels using sparse sensors is not feasible in realistic conditions where measurements may be inaccurate and the propagation conditions are uncertain. The goal is to use sensor power measurements to identify regions where the signal level exceeds, or falls below, a given threshold, and to provide a level of confidence in those determinations. In this work, a belief-based method using Dempster-Shafer analysis is developed, which can accommodate uncertainties due to the propagation conditions and sensor inaccuracies and combines evidence from different sensors to give a belief value for each state. The method is illustrated first for a simplistic, flat-earth model, then the impacts of parameter uncertainties, shadowing and sensor errors are incorporated. A real operating environment is emulated using a propagation prediction program, and it is demonstrated that the new approach is able to provide useful input to the spectrum management function and enables a sophisticated interpretation to support context-specific decision-making.
\end{abstract}

\section{Introduction}

The current approach to spectrum management requires the pre-assignment of channels in a given operating band based on anticipated requirements, to prevent interference. In a mobile, heterogeneous radio environment, where the demands on spectrum in a given location change with time, a more dynamic approach to spectrum management may be able to make more effective use of the band and support higher user throughput. The challenge is then to achieve an adequate representation of the spectrum occupancy in the band and geographic area of interest to support either manual or automated dynamic spectrum management. The spectrum management process is inevitably subjective, as different operational requirements necessitate a balance of risk and benefit in terms of interference and connectivity.

As described in [1], the 'radio environment map' (REM) is a database of spectrum information, which may include topography, spectrum policies, expected pathloss and applicable propagation models, locations and

Correspondence: tricia.willink@crc.gc.ca

Communications Research Centre, 3701 Carling Ave., P.O. Box 11490,

Station $\mathrm{H}$, Ottawa, Ontario K2H 8S2, Canada transmission characteristics of primary emitters. Alternative concepts of a REM consist of an actual map database that contains local signal power levels: this is also known as an 'available resource map' [2] and 'interference cartography' [3]. The database may be populated by information from the emitters themselves [2], from spectrum sensing devices [3] or some combination. In this work, the map is generated from measurements gathered by sensors spread throughout the environment. This must be achieved with as few sensors as possible because of the overhead required to collect and process the data [4].

In much of the theoretical work reported, pathloss between two points is modelled as a known function of their separation, but in real systems this is an average, not a true reflection of the actual pathloss. This was illustrated in [5] for the indoor environment; furthermore, the sensors were also shown to have errors in their reported measurements, which is an important factor that must be considered in REM generation.

Idealised models of the pathloss can be avoided in methods that use interpolation-type approaches in combining the collected sensor measurements to generate the REM. Power level interpolation methods such as Kriging have been proposed [6] and have been demonstrated with

\section{Springer}


densely and uniformly located sensors. Weighted interpolation techniques based on inverse distance weighting methods were considered in [7] and were applied to an indoor test environment. A spline-based spatial estimator of power spectral density (PSD) was developed in [8] for the case in which each emitter may transmit on several channels simultaneously. In [9] a PSD-estimator approach was proposed for similar scenarios, in which sensors cooperate in a distributed manner to estimate jointly the occupancy of several adjacent channels.

In a dynamic, complex environment, it is not realistic to attempt to create and maintain a real-time, highly detailed REM. To prevent damaging levels of interference at user terminals, it is sufficient to know whether the power level in the frequency range of interest exceeds, or does not exceed, some specified threshold. A lowresolution version of a REM is then appropriate, and the spectrum occupancy map (SOM) is proposed here to indicate whether or not the spectrum is considered to be 'in use.' This is a similar goal to that described in [9], although in that case, the technique estimates the local power levels. As a first stage, then, spectrum occupancy mapping should help the spectrum manager to focus on areas, both geographic and spectral, that need extra attention either because there is an increased risk of interference or there is an opportunity for additional assignments to users. In this process, the spectrum manager should be made aware of the quality of the information on which these decisions will be based.

The environment of interest consists of heterogeneous mobile users without fixed infrastructure. The aim is to segment the area into regions where the radio frequency (RF) power falls on either side of the specified threshold rather than to identify precisely the locations of emitters. In practice, the sparse and possibly unreliable information, as well as the variability in propagation, means that there cannot always be certainty in this decision. The SOM must therefore give the spectrum managers a confidence measure that the information available indicates that the defined threshold is exceeded, or not, and will be interpreted subjectively along with any other information or constraints available.

In this work, the Dempster-Shafer (DS) theory of evidence is proposed to deal with the many uncertainties in the problem of fusing data from RF sensors in realistic propagation environments. The DS theory combines evidence from different sources that support specified hypotheses; it allows for the possibility that the evidence does not distinguish between two or more hypotheses, and this allows the incorporation of uncertainties as well as ignorance (no relevant evidence).

The DS theory has been applied to related problems such as the classification of land cover [10] and segmentation of images [11]. It was used to evaluate the reliability of different types of sensors, with application to the problem of identifying vehicle types, in [12]. Related to the topic of spectrum access, a DS approach was used in [13] to identify the presence of a licensed user using the output of cyclostationarity-based detectors, assuming that the signal-to-noise ratios (SNRs) of the licensed user signal at the sensors were known. A similar problem was studied in [14], using energy detection at the sensors, and again the SNRs were assumed known, although gains were demonstrated over a soft information combining energy detection method even when those SNRs were not given. Similar assumptions were considered in [15] and [16] to detect the presence of a licensed user. A different problem was considered in [17], where the objective was to identify the location of a receiver in an indoor environment, and it was assumed that signal strength levels for different locations were known a priori, i.e. the radio environment map was already generated by prior measurements.

The SOM generation problem is described in Section 2, and the applicable DS theory is summarised in Section 3. To illustrate the spectrum mapping technique, an idealised scenario using a simple distance-based pathloss model and perfect sensing is used in Section 4.

In reality, the propagation environment is more complex and the sensors are not perfect. Delays in receiving the sensor measurements and a dynamic environment also introduce sensor errors. These uncertainties can be accounted for in the DS analysis and their impact is considered in Section 5. The selection of parameters is discussed in Section 6.

In Section 7, a commercial propagation prediction program is used to emulate propagation conditions in a real geographic region, which illustrates the challenges of applying typical modelling assumptions to the real world. This emulation is used to demonstrate the effectiveness of the DS-based SOM generation technique in a realistic environment, and the approach is compared to a power interpolation method. Finally, conclusions are presented in Section 8.

\section{The spectrum map problem}

Consider a geographic area with challenging propagation characteristics, i.e. with shadowing and large-scale variations in path loss due to topographic and man-made features. Within the area, there are $M \geq 1$ emitters whose locations and transmission characteristics are unknown.

There are $N$ sensors randomly located within the area that measure the local signal level. The sensor measurements are combined to determine the state of evidence, or belief, that the power level in a given location is above or below the specified threshold. Combining the belief functions over many locations produces the area's spectrum occupancy map. 
The resolution of the mapping is selected to satisfy the spectrum management function requirements: in the case of the low UHF band, for an area spanning tens of kilometres, it can be divided into cells with a radius of several hundred metres, with the cell centre taken as the representative location.

Combining the sensor measurements over a large area is computationally complex. To avoid this complexity, each cell in the area is considered independently, using only information from sensors in a prescribed local area. Thus, not all cells must be updated when new sensor measurements are collected; in a dynamic environment, only those cells whose changing status will impact the spectrum management function need to be re-evaluated.

In the work described herein, it is assumed that the sensor measurements are centralised at a fusion centre, where the SOM is generated and presented to the spectrum manager. However, as only local information is required to compute the occupancy belief at any arbitrary location, the proposed technique could readily be applied in a distributed way, as in [9]; but in this case, only a single information message from each sensor is required.

In computing the spectrum occupancy map, imperfections in the data collection system must be taken into account. The sensors are randomly located, and some regions may have inadequate coverage. The measurements themselves may be inaccurate due to sensor errors or to time delays in their transmission to the fusion centre, and the locations of the sensors may not be accurately known. The operating environment is challenging: pathloss characteristics may be highly localised, and the correlation distance of shadowing caused by natural and man-made obstacles may vary widely over the area. Local variations in terrain may result in conflicting evidence provided by sensors in close proximity.

The SOM is required to support the spectrum management function to make context-specific decisions that necessitate balancing the risk of interference with demand for spectrum access. The aim of the proposed technique is to produce a SOM that provides useful input to the spectrum manager in spite of the challenges of a real scenario. The SOM should indicate where support for either of the two states (above or below the power threshold) is weak or strong, as well as where there is insufficient coverage to provide reliable information.

\section{Dempster-Shafer theory}

The Dempster-Shafer or DS theory is an evidential reasoning technique that provides an approach in dealing with uncertainty in data fusion. It is usually formulated in terms of belief functions, introduced by Shafer in [18], and integrated with similar ideas from Dempster developed in the 1960s, e.g. [19].

There are numerous points of view regarding the DS theory, and there have been debates regarding its interpretation, particularly in the late 1980s and 1990s, for example, in $[20,21]$ and responses thereto. It is sometimes interpreted in probabilistic terms (see Shafer [22] and Dempster [23] for more recent discussions and perspectives). However, more recently, it was claimed by Smets [24] that the DS model is 'completely unrelated to the probability model'. It was shown in [24] and in the subsequent work that a model for the fusion of evidence, the transferable belief model (TBM), could be derived without relying on a concept of probability. From the point of view of the problem addressed herein, the TBM and DS theory are effectively equivalent. The non-probabilistic interpretation is used here: as discussed in the introduction, the question of interest here relates to confidence, not probability.

A comparison of the Bayesian and DS approaches in [25] illustrates that the two techniques can lead to similar results but that a well-defined formulation of probabilities, conditioning and priors is required in the Bayes method, whereas in the DS theory, conditioning is incorporated in the belief functions with no prior knowledge needed. A discussion of the similarities and differences between the two approaches is also given in [26]. In the examples given in Section 1, in particular [13-17], the problems could have been formulated similarly using a Bayesian approach because the probabilities used to define the hypotheses were well-defined. As noted in [27], the DS theory allows the problem to be formulated to reflect the state of knowledge, rather than physics, which is particularly applicable in this work, as there are too many unknowns (emitter power, pathloss and locations) to be able to define precisely the actual state. The DS theory also supports the use of 'unknown' as a state, which is also useful in the current problem.

The relevant DS theory is given in the remainder of this section; for more detail on the mathematical basis, see the references cited above. The DS theory is applied to the idealised spectrum map problem first to illustrate how the evidence is generated and combined. The strength of the DS theory is in incorporating the effects of the uncertainties and measurement inaccuracies that are inevitable in real applications: these are considered in the later sections.

\subsection{Evidence}

At each sensor $j$, evidence is collected that may provide support for or against possible states $\omega_{i}$, which are the elements of the state space or frame of discernment, $\Omega$. The function $m$ is known as the basic belief assignment, given 
by the mapping of the power set $m: 2^{\Omega} \rightarrow[0,1]$ (see for example [12]), as defined by

$$
\sum_{A \subseteq \Omega} m(A)=1 .
$$

Unlike in probability theory, a mass can be ascribed to any subset of $\Omega$, not just to its elements. Thus, the basic belief mass (bbm) $m\left(\left\{\omega_{1}, \omega_{2}\right\}\right)$ is defined to address the case where evidence points to two elements $\omega_{1}$ and $\omega_{2}$ but does not distinguish between them.

\subsection{Evidence combining}

The rule of combination [23,24] is applied to combine the belief functions obtained from independent sensors. This can be written in terms of the bbms as, for sensors $i$ and $j$ and $A \subseteq \Omega, A \neq \varnothing$,

$$
m(A)=\left(m_{i} \oplus m_{j}\right)(A)=\frac{\sum_{X \cap Y=A} m_{i}(X) m_{j}(Y)}{1-\sum_{X \cap Y=\varnothing} m_{i}(X) m_{j}(Y)}
$$

and $m(\varnothing)=0$.

The term $\sum_{X \cap Y=\varnothing} m_{i}(X) m_{j}(Y)$ in the denominator of (2) is the conflict between $m_{i}$ and $m_{j}$. The application of normalisation in the selection of two independent hypotheses was discussed in [28]. In the current work, the hypotheses are dependent: the power is either above or below the specified threshold, hence normalisation leads to the possibility of a consistent metric for assessing the belief that the spectrum in a given cell is occupied.

\subsection{Belief and plausibility}

For each piece of evidence, $E_{k} \subseteq \Omega$, the bbm $m\left(E_{k}\right)$ describes the associated belief. For some $A \subseteq \Omega$, $\operatorname{bel}\{A\}$ is the degree of belief that the true state belongs to $A$, and is determined by accumulating all evidence that supports the proposition $A$, i.e.

$$
\operatorname{bel}\{A\}=\sum_{E_{k} \subseteq A} m\left(E_{k}\right) .
$$

The normalisation in (2) also gives bel $\{\varnothing\}=0$ and $\operatorname{bel}\{\Omega\}=1$.

Another quantity, the plausibility, is defined to describe the maximum potential support for a proposition, i.e. the combination of evidence that does not contradict it,

$$
\operatorname{pl}\{A\}=\sum_{E_{k} \cap A \neq \varnothing} m\left(E_{k}\right)=1-\operatorname{bel}\{\bar{A}\}
$$

where $\bar{A}$ is the complement of $A$. The possibility of uncertainty is then allowed by $\operatorname{pl}\{A\}>\operatorname{bel}\{A\}$.

The application of the DS theory to the spectrum mapping problem will be described first for the simplest case of no uncertainties in measurements or the propagation model.

\section{Spectrum occupancy map: ideal case}

Consider a geographic area of dimension $L \times L$, divided into hexagonal cells with centres separated by $d_{\text {hex }}$. An unknown number, $M$, of emitters are operating in the area at unknown locations. The emitters are assumed to have omni-directional antennas, and lower and upper bounds, $P_{\mathrm{e}, \min }$ and $P_{\mathrm{e}, \mathrm{max}}$, respectively, can be specified for their effective powers based on the applicable emission policies. Multiple emitters in a small area will not generally be separable, so their combined power should be used as the upper limit. In this ideal case, flat-earth propagation is assumed, i.e. at a distance $d$ from an emitter of power $P_{\mathrm{tx}}$, the received power is given by

$$
P_{\mathrm{rx}}=P_{\mathrm{tx}}-L\left(d_{0}\right)-10 \alpha \log \frac{d}{d_{0}}
$$

where $d_{0}$ is a reference distance, $L\left(d_{0}\right)$ is the pathloss from the emitter to the distance $d_{0}$, which is dependent on the signal wavelength, and $\alpha$ is the pathloss exponent. In this work, the effective transmission power $\tilde{P}_{\mathrm{tx}}=P_{\mathrm{tx}}-L\left(d_{0}\right)$ is defined for convenience, yielding

$$
P_{\mathrm{rx}}=\tilde{P}_{\mathrm{tx}}-10 \alpha \log \frac{d}{d_{0}} .
$$

Here, $d_{0}$ will be selected to be less than the cell radius.

The SOM is generated using the measurements obtained at $N$ omni-directional sensors located in the $L \times L$ area. The sensors are in different cells and for this ideal case, they are assumed to measure the received signal power in their cells perfectly. Both sensors and emitters are assumed, for now, to be located in the centre of their cells.

The information generated by fusing the sensor data using the DS theory is the degree of belief that the total signal power in any given cell exceeds the threshold $P_{\text {thresh }}$. Combining the sensor measurements over a large area, for many channels in the frequency band, is computationally complex. To manage this complexity, each cell in the area is considered independently. This approach provides the advantage that cells where there is little discrimination between hypotheses $A$ and $B$ can be updated more frequently as new data become available, compared to cells where the distinction is clear and unlikely to change with small changes in sensor evidence. This means that in a dynamic environment, only those cells that might impact the spectrum management function need to be updated, leading to a more efficient tracking than if all cells must be updated immediately at every time interval.

The frame of discernment, $\Omega$, is defined as $\Omega=\{A, B\}$ where $A$ and $B$ are the two hypotheses 'above $P_{\text {thresh }}$ ' and 'below $P_{\text {thresh }}$ ', respectively. The set of all subsets of $\Omega$ is $\{\varnothing, A, B,\{A, B\}\}$; hence, $m(A)$ represents the evidence that $P_{\text {thresh }}$ is exceeded, $m(B)$ represents the evidence that 
$P_{\text {thresh }}$ is not exceeded, and $m(\{A, B\})$ represents the evidence that cannot discriminate. In this case, as $A$ and $B$ are opposing hypotheses, $m(\varnothing)=0$.

\subsection{Evidence computation}

The aim of the evidence computation is to determine, for each cell in the $L \times L$ area, the basic belief masses for the two hypotheses, $A$ and $B$, denoting power levels above and below the specified threshold. Consider cell $c_{i}$ : unless there is a sensor in $c_{i}$, the true power level is unknown, and the information available from sensors in the neighbourhood must be used to develop a belief model for that cell.

Consider sensor in cell $c_{j}$, a distance $d_{c}$ from $c_{i}$. The power level measured, which is a combination of signals received from all emitters, is $P_{s, j}(\mathrm{dBm})$. The measurements obtained by this sensor can be used to determine limits on the range of powers that could be observed in cell $c_{i}$.

To estimate the range of powers that might be observed in cell $c_{i}$, based on data from the sensor in cell $c_{j}$, consider a single emitter with effective transmission power $\tilde{P}_{\mathrm{e}}$ located at a distance $d_{x}$ from $c_{i}$ and an estimated distance $\hat{d}_{j, e}\left(\tilde{P}_{\mathrm{e}}\right)$ from the sensor, which can be computed from (6) as

$$
\hat{d}_{j, e}\left(\tilde{P}_{\mathrm{e}}\right)=d_{0}\left(10^{\left(\tilde{P}_{\mathrm{e}} / P_{s, j}\right) / 10}\right)^{\frac{1}{\alpha}} .
$$

It can readily be shown that the minimum power in cell $c_{i}$ is achieved with a single emitter, located along a straight line joining $c_{i}$ and $c_{j}$, as in Figure 1a with $d_{x}=\hat{d}_{j, e}\left(\tilde{P}_{\mathrm{e}}\right)+d_{c}$, and is given by

$$
P_{i, j, \min }=\min _{\tilde{P}_{\mathrm{e}}}\left(P_{s, j}+10 \alpha \log _{10}\left(\frac{\hat{d}_{j, e}\left(\tilde{P}_{\mathrm{e}}\right)}{d_{x}}\right)\right) .
$$

This is minimised when $\tilde{P}_{\mathrm{e}}=\tilde{P}_{\mathrm{e}, \mathrm{min}}$, which is the effective minimum transmitted power, corrected for the short distance loss $L\left(d_{0}\right)$.

Using (6), the maximum power in cell $c_{i}$ is given by

$$
P_{i, j, \max }=\max _{\tilde{P}_{\mathrm{e}}}\left(P_{s, j}+10 \alpha \log _{10}\left(\frac{\hat{d}_{j, e}\left(\tilde{P}_{\mathrm{e}}\right)}{d_{x}}\right)\right)
$$

where $d_{x}=\left|d_{c}-\hat{d}_{j, e}\left(\tilde{P}_{\mathrm{e}}\right)\right|$, as illustrated in Figure 1b,c. This approach combines all nearby emitters into a single effective emitter.

Both the minimum and maximum powers may be fairly loose limits. It has been found that the actual values are not critical, if the sensor density is sufficiently large, due to the combining of evidence. If, for example, the upper limit is grossly over-estimated, the evidence from other sensors will either contradict this maximum, or it will support that the cell power is much greater than the threshold $P_{\text {thresh }}$, which is all the information that is required for aiding the spectrum manager.

Finally, if $i=j$ then the power level in that cell is given by the sensor value, i.e.

$$
P_{i, j \min }=P_{i, j, \max }=P_{s, j}
$$

The upper and lower limits of the possible signal power level in cell $c_{i}$ are used to determine the bbms using the principle of insufficient reason, or indifference (Bernoulli, Ars conjectandi, 1713). This says that, absent any other information, equal weight must be given to each possibility. A uniform distribution of evidence over the range $P_{i, j \text { min }} \leq P_{c, i}<P_{i, j \text {, max }}$ means that the bbms for cell $c_{i}$ obtained from sensor $j$ are, for $i \neq j$

$$
\begin{aligned}
m_{i, j}(A) & =r \cdot\left(\frac{P_{i, j, \max }-P_{\text {thresh }}}{P_{i, j, \max }-P_{i, j, \mathrm{~min}}}\right)^{+} \\
m_{i, j}(B) & =r \cdot\left(\frac{P_{\text {thresh }}-P_{i, j, \mathrm{~min}}}{P_{i, j, \max }-P_{i, j, \mathrm{~min}}}\right)^{+} \\
m_{i, j}(\{A, B\}) & =(1-r)
\end{aligned}
$$

where $(a)^{+}=a$ if $a \geq 0$ and 0 otherwise, and $r$ is a measure of the reliability assigned to the information from sensor $j$ for cell $c_{i}$.

When the cell contains a sensor, i.e. $i=j, m_{i, j}(A)=1$

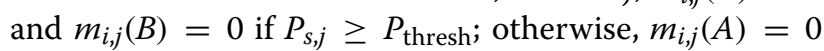
and $m_{i, j}(B)=1$; and $m_{i, j}(\{A, B\})=0$ in both cases.

In this work, a simple distance-based reliability is used, given by

$$
\begin{aligned}
r & =\left(\frac{d_{\text {hex }}}{d_{c}}\right)^{\beta} & & d_{c}<D_{\mathrm{s}} \\
& =0 & & d_{c} \geq D_{\mathrm{s}}
\end{aligned}
$$

where $\beta>0$ is a parameter, and $D_{\mathrm{s}}$ is the maximum distance for which sensors' evidence is incorporated. This is similar to the distance-based weighting used in the power interpolation methods considered in [7]. The selection of these parameters is discussed in Section 6.

\subsection{Combining evidence for all sensors}

Sensors are in different cells, therefore the evidence obtained from them is independent, and can be combined using (2) and the chain rule for cell $c_{i}$, as follows:

$$
\begin{aligned}
m_{i}(A) & =m_{i, 1}(A) \\
m_{i}(B) & =m_{i, 1}(B) \\
m_{i}(\{A, B\}) & =m_{i, 1}(\{A, B\})
\end{aligned}
$$


(a)

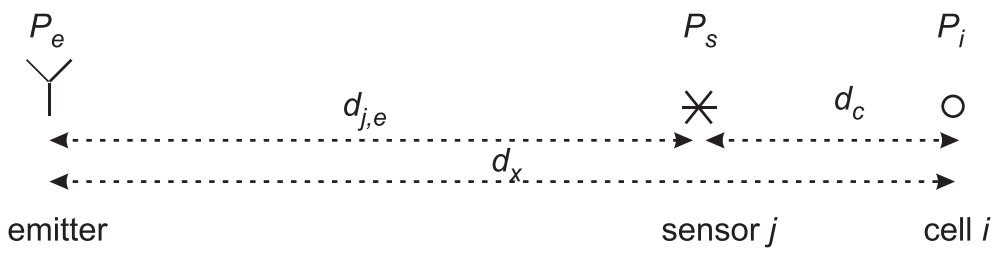

(b)

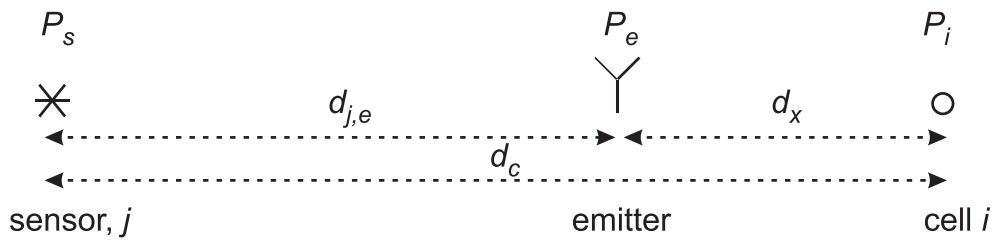

(c)

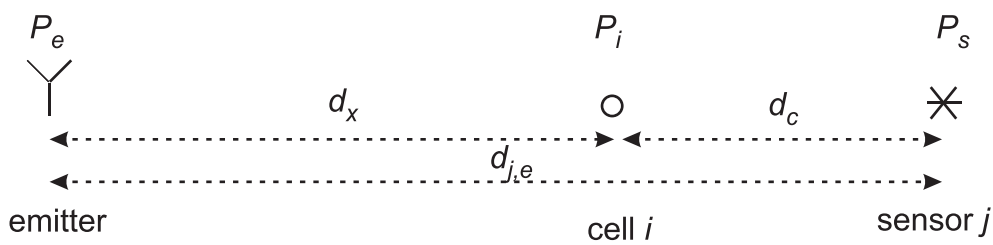

Figure 1 Illustration of emitter configurations for power range in cell $\boldsymbol{c}_{\boldsymbol{i}}$. (a) Minimum power in cell $c_{i}$. (b) Maximum power in cell $c_{i}, d_{j, e}<d_{c}$. (c) Maximum power in cell $c_{i}, d_{j, e}>d_{c}$.

$$
\begin{aligned}
& \text { for } j=2, \ldots, N \\
& K=1-m_{i}(A) \cdot m_{i, j}(B)-m_{i}(B) \cdot m_{i, j}(A) \\
& m_{A}=\frac{1}{K}\left(m_{i}(A) \cdot m_{i, j}(A)+m_{i}(A) \cdot m_{i, j}(\{A, B\})\right. \\
&\left.+m_{i}(\{A, B\}) \cdot m_{i, j}(A)\right) \\
& m_{B}= \frac{1}{K}\left(m_{i}(B) \cdot m_{i, j}(B)+m_{i}(B) \cdot m_{i, j}(\{A, B\})\right. \\
&\left.+m_{i}(\{A, B\}) \cdot m_{i, j}(B)\right) \\
& m_{A B}= \frac{1}{K} m_{i}(\{A, B\}) \cdot m_{i, j}(\{A, B\}) \\
& m_{i}(A)= m_{A}, \quad m_{i}(B)=m_{B}, \quad m_{i}(\{A, B\})=m_{A B}
\end{aligned}
$$

end

\subsection{Power level belief and plausibility}

The belief function for cell $c_{i}$ is computed from the combined bbms using (3), which gives

$$
\begin{aligned}
\operatorname{bel}_{i}\{A\} & =\kappa \cdot m_{i}(A) \\
\operatorname{bel}_{i}\{B\} & =\kappa \cdot m_{i}(B)
\end{aligned}
$$

where $\kappa$ is a normalisation factor such that $\operatorname{bel}\{A\}+$ $\operatorname{bel}\{B\}+\operatorname{bel}\{A, B\}=1$.

Similarly, the plausibility function for cell $c_{i}$ is, from (4)

$$
\begin{aligned}
\operatorname{pl}_{i}\{A\} & =1-\kappa \cdot m_{i}(B) \\
\operatorname{pl}_{i}\{B\} & =1-\kappa \cdot m_{i}(A)
\end{aligned}
$$

which means that $\operatorname{pl}_{i}\{A\}-\operatorname{bel}_{i}\{A\}=\operatorname{pl}_{i}\{B\}-\operatorname{bel}_{i}\{B\}=$ $\kappa \cdot m_{i}(\{A, B\})$, which is a metric for the residual uncertainty.

\subsection{Ideal case results}

An example of the application of the DS analysis is given here for the idealised case; the impact of more realistic assumptions will be explored in the subsequent section.

Figure 2a shows the 'ground truth' for this example. The map covers an area of $20 \times 20 \mathrm{~km}$ and is divided into hexagonal cells with centres on a grid at spacings of $d_{\text {hex }}=$ $500 \mathrm{~m}$. The reference distance used was $d_{0}=100 \mathrm{~m}$. There are $M=3$ emitters in this example, randomly placed at least $8 \mathrm{~km}$ apart, operating at $300 \mathrm{MHz}$ with transmit powers randomly selected from the uniform distribution between 15 and $25 \mathrm{~W}$. The number, location and powers of the emitters are not known to the processing algorithm. In this idealised example, the flat-earth propagation model is used to determine the ground truth, using pathloss exponent $\alpha=3$ in (6).

There are $N=50$ sensors randomly located within the area, with the restriction that no cell contains more than one sensor, nor a sensor and an emitter. The sensors are assumed to be located at the centre of their containing cells and to detect the signal power exactly.

The objective of the analysis is to use the sensed information to determine whether the signal power in 


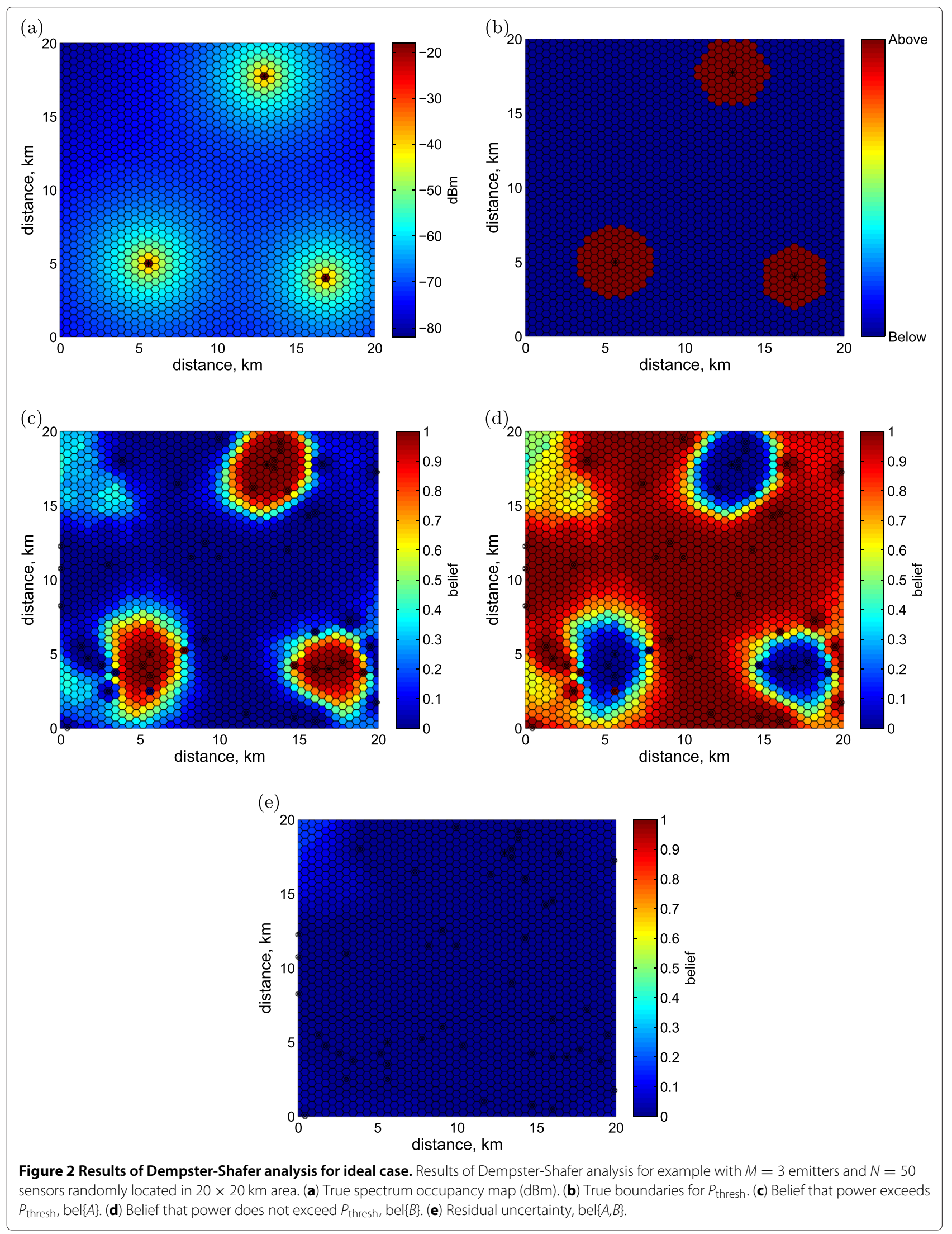


each cell is less than or greater than the threshold of $P_{\text {thresh }}=-60 \mathrm{dBm}$. Figure $2 \mathrm{~b}$ shows the true boundaries at this threshold.

The DS analysis has been applied to this problem as described above. The reliability $r$ is computed using (12), with a maximum distance $D_{\mathrm{s}}=10 \mathrm{~km}$ and $\beta=0.5$; the justification for these parameters is discussed in Section 6. The resulting belief functions $\operatorname{bel}\{A\}$ and $\operatorname{bel}\{B\}$ are shown in Figure $2 \mathrm{c}, \mathrm{d}$. The residual uncertainty given by bel $\{A, B\}$ is shown in Figure $2 \mathrm{e}$. As $\operatorname{bel}\{A, B\} \approx 0$ over most of the area, the plausibilities $\operatorname{pl}\{A\}$ and $\operatorname{pl}\{B\}$ are approximately equal to the complements of $\operatorname{bel}\{A\}$ and $\operatorname{bel}\{B\}$ and are not shown.

In this ideal case, the DS analysis shows, with a high degree of belief, the regions where the signal power is high. The regions of high signal power around the three emitters can be clearly identified, and the belief that the power exceeds (Figure 2c) or does not exceed (Figure 2d) the threshold decreases or increases, respectively, as the distance from those emitters increases.

As expected, the most confidence, for both sets $A$ and $B$, is obtained in the cells that contain a sensor, and, if there is no conflicting evidence from other sensors, there are slowly decreasing circles of belief as the distance from the sensor increases. The neighbouring cells also show a high degree of confidence - over $90 \%$ of the cells adjacent to sensorcontaining cells have correct beliefs exceeding 0.9. The beliefs decrease further from the sensor cells, such that $70 \%$ of cells at a two-cell distance from the sensor cells have correct beliefs exceeding 0.9 - this is expected as well, as the information provided by the sensors has less direct relevance.

In the regions where there are insufficient sensors, the evidence is weak and/or contradictory, and the beliefs tend to neutral, i.e. around 0.5 . This lack of discrimination is seen in the north-west and south-west regions where the evidence provided by the sensors in range is nearly equally divided between sets $A$ and $B$. With very few nearby sensors, the uncertainty increases, such as in the north-west region, as shown in Figure 2e. Overall, the density of the sensors in this example is sufficient to achieve a low degree of uncertainty, with $90 \%$ of the cells having a residual uncertainty of 0.02 or less.

The evidence provided by the different sensors is illustrated in Figure 3 for the cell located at the centre of the map, $(10,10) \mathrm{km}$. For each sensor $j$ within range $D_{\mathrm{s}}$, the computed range $\left[P_{i, j, \mathrm{~min}}, P_{i, j, \mathrm{max}}\right]$ is indicated and coloured by its reliability, $r$, which is a function of its distance from the cell of interest. The threshold $P_{\text {thresh }}$ is marked by a dark line. The bbm for each sensor in range is computed using (11a): of the 31 sensors within range $D_{\mathrm{s}}$, 19 have $P_{i, j, \max }<P_{\text {thresh }}$, resulting in $m(A)=0$, i.e. their evidence only supports the hypothesis that the power level is below the given threshold. The other 12 sensors have $m(A)>0$ and $m(B)>0$. Without the reliability parameter, i.e. with $r=1$ for all sensors, the evidence from the 19 sensors with $P_{i, j \text { max }}<P_{\text {thresh }}$ would discount that from the other 12 in the combination process (Section 3.2), even though the latter may, in fact, be more trustworthy.

Figure 4 shows the cumulative distribution functions (CDFs) of belief functions bel $\{A\}$ and bel $\{B\}$, each combined over ten random scenarios with the number of

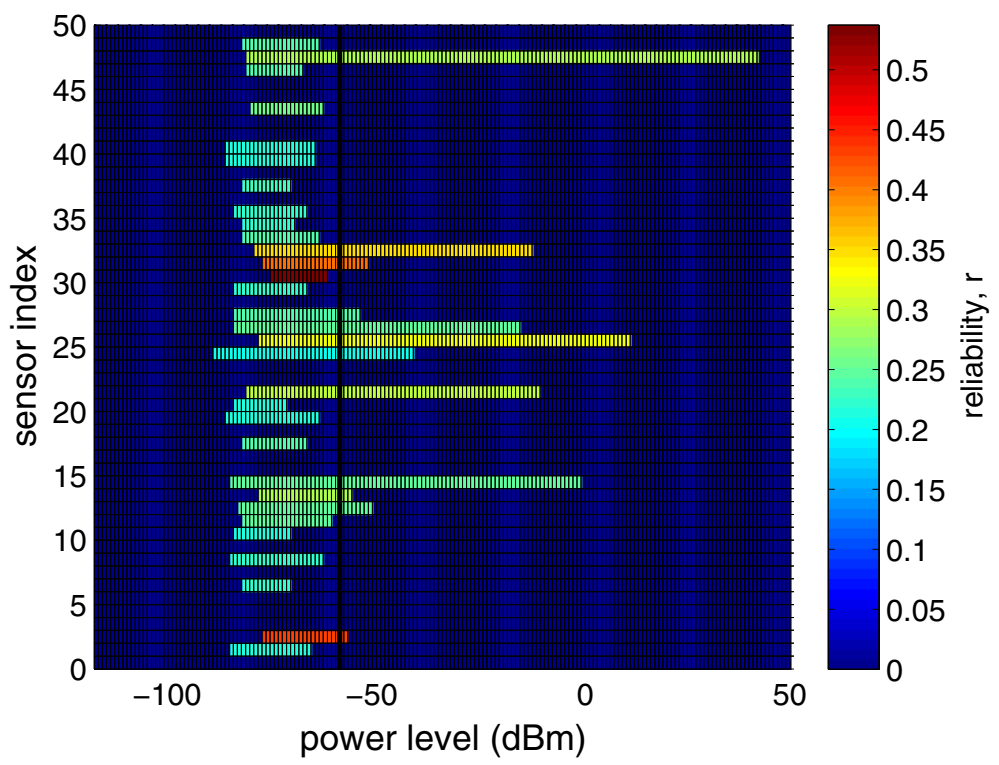

Figure 3 Reliabilities for sensor evidence, cell at $\left(\mathbf{1 0 , 1 0 )} \mathbf{k m}\right.$. Line indicates $P_{\text {thresh }}=-60 \mathrm{dBm}$. 


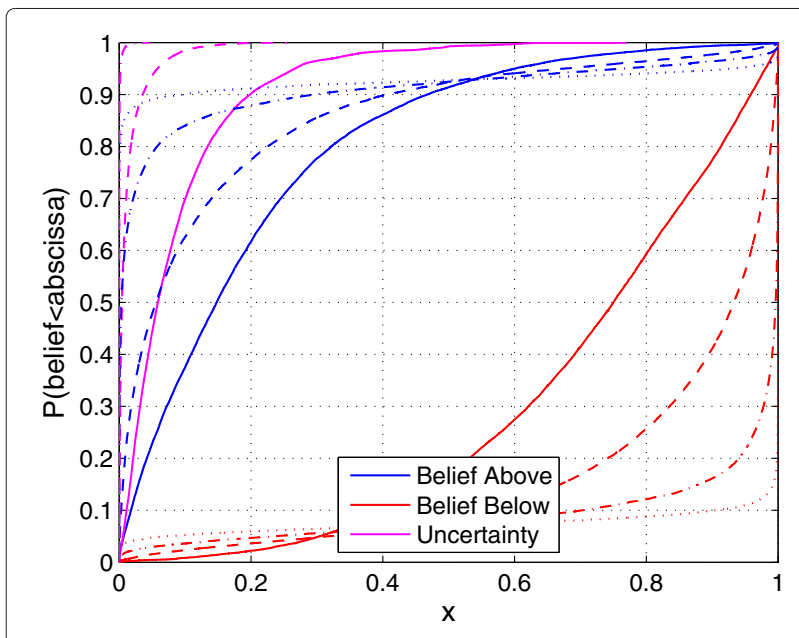

Figure $4 \mathrm{CDFs}$ of belief functions for different numbers of sensors. CDFs of belief functions for $N=25$ (solid lines), $N=50$ (dashed lines), $N=100$ (dash-dotted lines) and $N=200$ (dotted lines).

emitters $M$ uniformly distributed between 1 and 4, each with a random power between 15 and $25 \mathrm{~W}$. The CDFs for $N=25,50,100,200$ sensors are shown. Approximately $11 \%$ of cells have true power levels above the threshold. For a large number of sensors, bel $\{A\}$ and bel $\{B\}$ are dominantly near zero or one, and there is no measurable uncertainty. As the number of sensors decreases, there is less evidence available and a lower probability of a nearby sensor that provides high value evidence; hence, the belief functions become less polarised.

In this idealised model, the boundaries of the regions with power levels above and below the threshold are smooth and clearly defined, so results showing the polarised behaviour of the belief functions are considered successful. However, as will be seen in the next section, models that take into account more challenging propagation conditions do not produce such well-structured regions. The advantage of the DS belief function approach is more evident in these more realistic models, as information from the sensors cannot be expected to reproduce random features such as shadow fading; rather, the objective is to determine a level of confidence, or belief, in each possible hypothesis. Uncertainties such as imperfect estimation of propagation parameters and sensor inaccuracies introduce a further degree of randomness, and the use of the DS theory provides a more nuanced interpretation of the sensed information than is available from a conventional probabilistic approach.

\section{Non-ideal models and uncertainties}

In Section 4, standard assumptions were made about the propagation environment, namely, that it follows a welldefined model and that the parameters of that model are known. The model parameters widely used in simulated environments are obtained from a statistical analysis of measurements obtained in a variety of locations and are not, in fact, accurate representations of individual radio paths. For example, terrain or man-made features in one area will affect the local pathloss and shadowing parameters. This means that, in practice, the characteristics of the radio environment measured by sensors are not accurately known to the processing algorithm. The effects of imperfect knowledge of pathloss parameters, shadowing and sensor errors are investigated in this section, using simulated propagation environments.

\subsection{Pathloss errors}

Pathloss is typically modelled as an exponential decay, as in (6), where the exponent $\alpha$ varies over a range of approximately 2 to 5 [29], depending on the density of scattering objects, e.g. buildings and trees. The value of $\alpha$ is estimated from many measurements in an area, based on the average signal power decay with distance. It is typically taken to be uniform over a given area, although this is unlikely to be true, especially as the size of the area increases. Nonetheless, in this section, the pathloss exponent $\alpha$ is assumed to be constant over the whole area, and the effect of having an incorrect estimate, $\hat{\alpha}$, is considered.

The estimate $\hat{\alpha}$ is used in the computation of evidence, specifically in estimating the distance that an emitter must be from the current cell, (6), and thereby the corresponding minimum and maximum power levels, (8) and (9).

Figure 5 shows the belief functions bel $\{A\}$ for an estimated pathloss exponent, $\hat{\alpha}=2.5$ and 3.5. The simulation parameters are the same as in the ideal example in Section 4, using the same locations for the $M=3$ emitters and $N=50$ sensors, to allow a direct comparison.

The figure shows quite a marked difference in belief function resulting from under- and overestimating the pathloss exponent. The results indicate that overestimating the pathloss parameter $\alpha$ tends (in this idealised scenario) to identify the locations of the emitters but does not provide a reliable indication of the regions separated by the power threshold. Note that if the sensor distribution is sparse, this approach risks failing to identify the existence of an emitter. When the pathloss exponent is underestimated, there is a larger region around the threshold boundaries where there is a lack of discrimination resulting from conflicting evidence, i.e. the belief function is close to neutral, i.e. $\operatorname{bel}\{A\} \approx \operatorname{bel}\{B\}$.

\subsection{Shadowing and sensing inaccuracies}

Shadowing is a large-scale pathloss effect resulting from obstacles blocking the propagation of signal energy. It is measured as the variation of the measured pathloss about the underlying trend, for example, (6), and is typically 

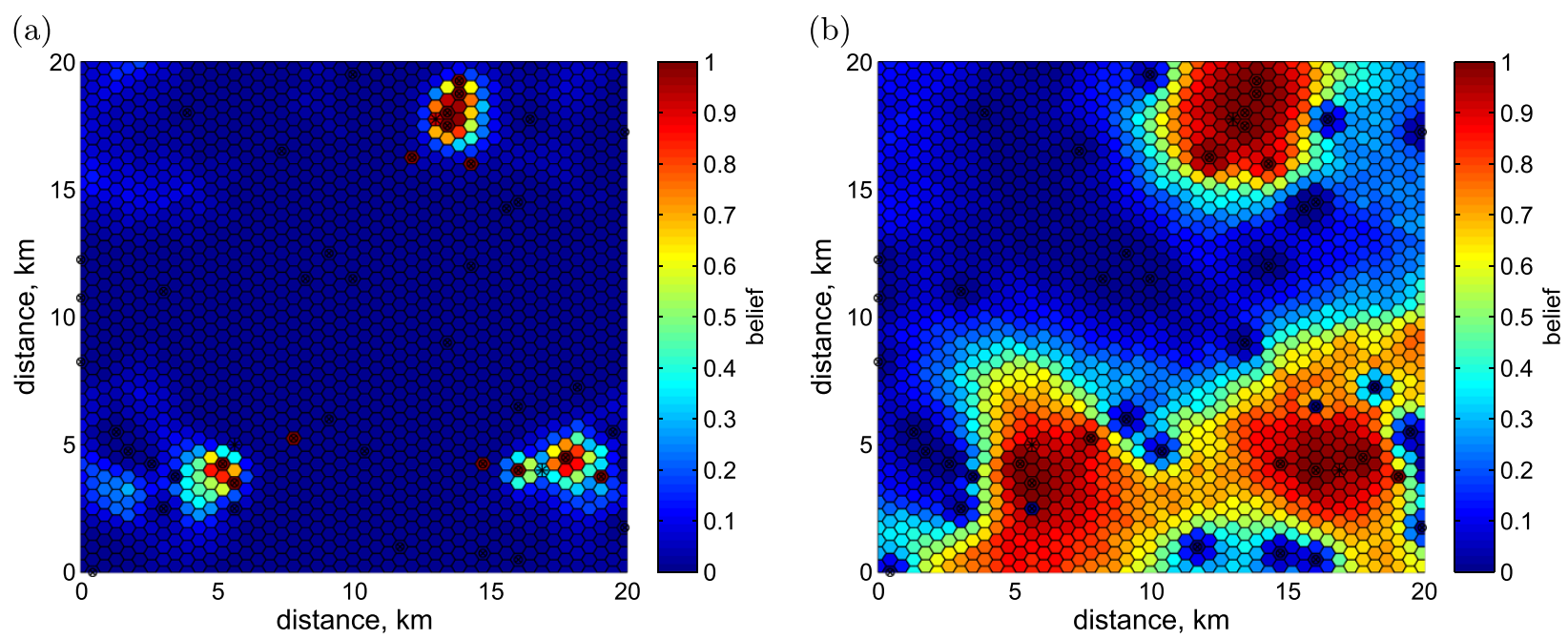

Figure 5 Belief for incorrect pathloss exponents. Belief that power exceeds $P_{\text {thresh }}$ for example with $M=3$ emitters and $N=50$ sensors in $20 \times$ $20 \mathrm{~km}$ area, with incorrect estimate of pathloss exponent (true $\alpha=3$ ). Threshold $P_{\text {thresh }}=-60 \mathrm{dBm}$. (a) $\hat{\alpha}=3.5$. (b) $\hat{\alpha}=2.5$.

modelled as a lognormal process. The impact of the shadowing is to introduce an uncertainty into the relevance of the sensor measurements, as the path between the emitter and the cell of interest will have a different shadow loss than the paths from the emitter to each sensor. This is also a challenge for modelling shadowing over an area, as the pathloss is correlated in space. The standard statistical shadow model, which applies between two points [29], does not represent the spatial correlation effects of shadowing. A spatial loss field method, described in the Appendix, is used here.

An additional source of uncertainty is the sensors themselves. To this point, these sensors have been assumed to be perfect, i.e. to provide an accurate value of the cell power, whereas in reality there may be sensor bias and other errors. The assumption has also been made that the sensor is located in the centre of the cell. The sensor error is modelled in two parts: an error uniformly distributed over $[-3,3] \mathrm{dB}$ to represent the sensing inaccuracy and a random variation in distance from each emitter, uniformly distributed over $\left[-d_{\text {hex }}, d_{\text {hex }}\right] ; 90 \%$ of these errors lie between $-1 \mathrm{~dB}$ and $1 \mathrm{~dB}$. This sensor error term could also include the effect of delays in receiving the sensor measurements, resulting from congestion in the network or mobility in the environment.

The effect of these uncertainties is to expand the possible ranges of power in the cell of interest used in (11a) to compute the bbms for the hypotheses ' $\mathrm{A}$ ' and ' $\mathrm{B}$.' No model is assumed for the uncertainties in the DS implementation; instead, limits on the pathloss variations are defined. For the shadowing component, the pathloss is assumed to be in the range $[-\Sigma, \Sigma]$, and the sensor error is in the range $[-\Phi, \Phi]$.
As in (8), the minimum power in the cell $c_{i}$ is still achieved for the smallest effective transmitted power, $\tilde{P}_{\mathrm{e}, \min }$, but now an additional pathloss due to the worst case shadowing and sensor inaccuracies must be included. This leads to

$$
P_{i, j \text { min }}=P_{s, j}+10 \alpha \log _{10}\left(\frac{\hat{d}_{j, e}\left(\tilde{P}_{e, \min }\right)}{\hat{d}_{j, e}\left(\tilde{P}_{\mathrm{e}, \min }\right)+d_{c}}\right)-\Sigma-\Phi .
$$

Similarly, the upper power bound in cell $c_{i}$ is found from (9), with an additional term to account for reduced pathloss because of the worst case uncertainties in received power, i.e.

$$
P_{i, j, \max }=P_{s, j}+10 \alpha \log _{10} \max _{\tilde{P}_{\mathrm{e}}}\left(\frac{\hat{d}_{j, e}\left(\tilde{P}_{\mathrm{e}}\right)}{\left|d_{c}-\hat{d}_{j, e}\left(\tilde{P}_{\mathrm{e}}\right)\right|}\right)+\Sigma+\Phi .
$$

No attempt is made to consider the fading correlations between cells. This would require the estimation of another parameter, the correlation distance, which is, in reality, quite localised although it is typically characterised over a large area. Furthermore, it contradicts the goal of addressing each cell independently.

The simulations in this section address only the imperfect knowledge due to shadowing; the pathloss exponent is given by $\alpha=3$, and the estimated value is also $\hat{\alpha}=3$. The combination of inaccurate pathloss estimates has a similar effect on these results as shown in Section 5.1.

Figure 6a shows the ground truth for the same configuration of emitters and sensors as in the example above, but now, by incorporating a random shadowing field, 

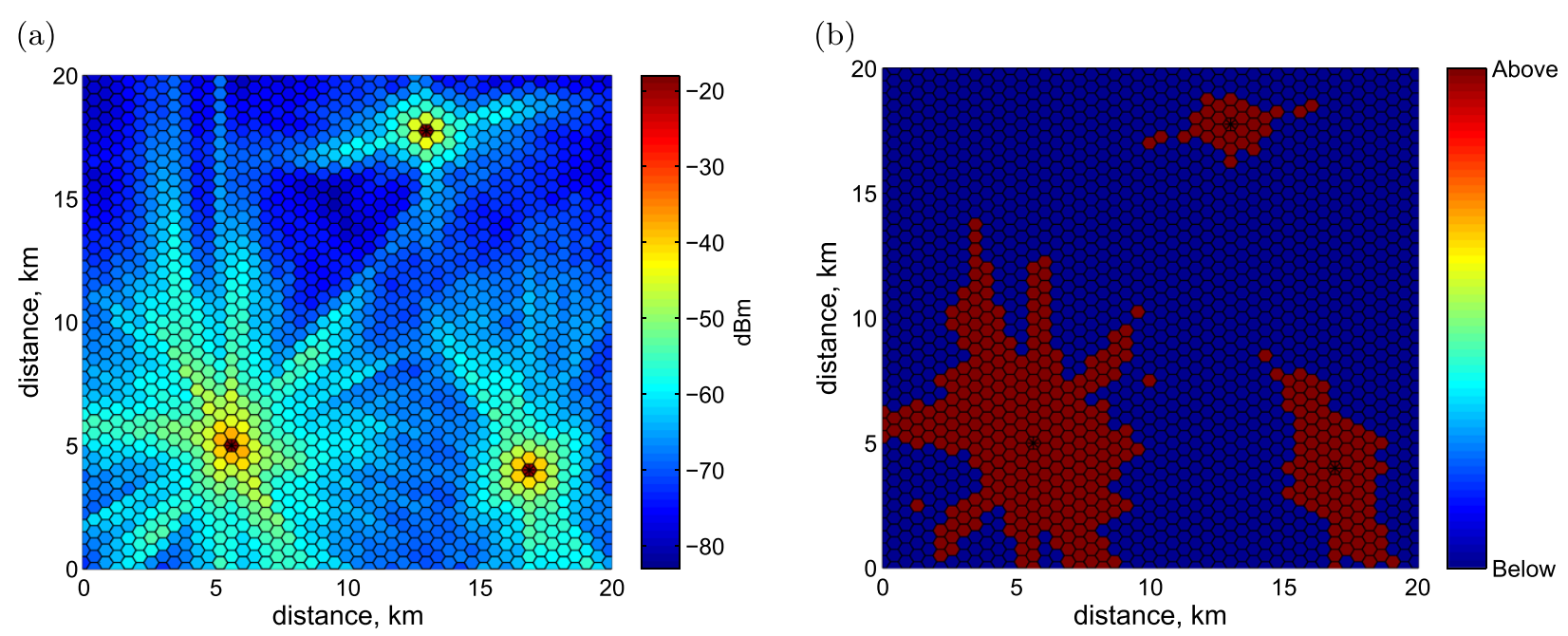

(c)
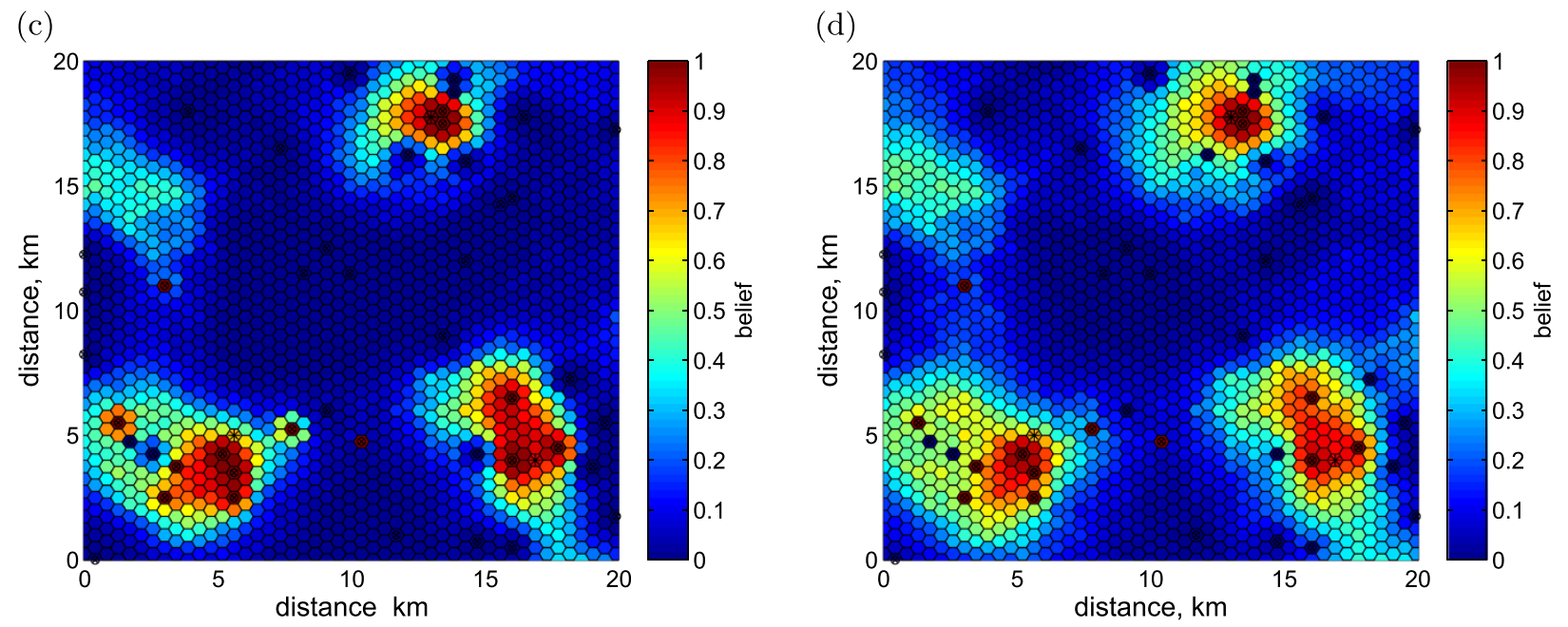

(e)
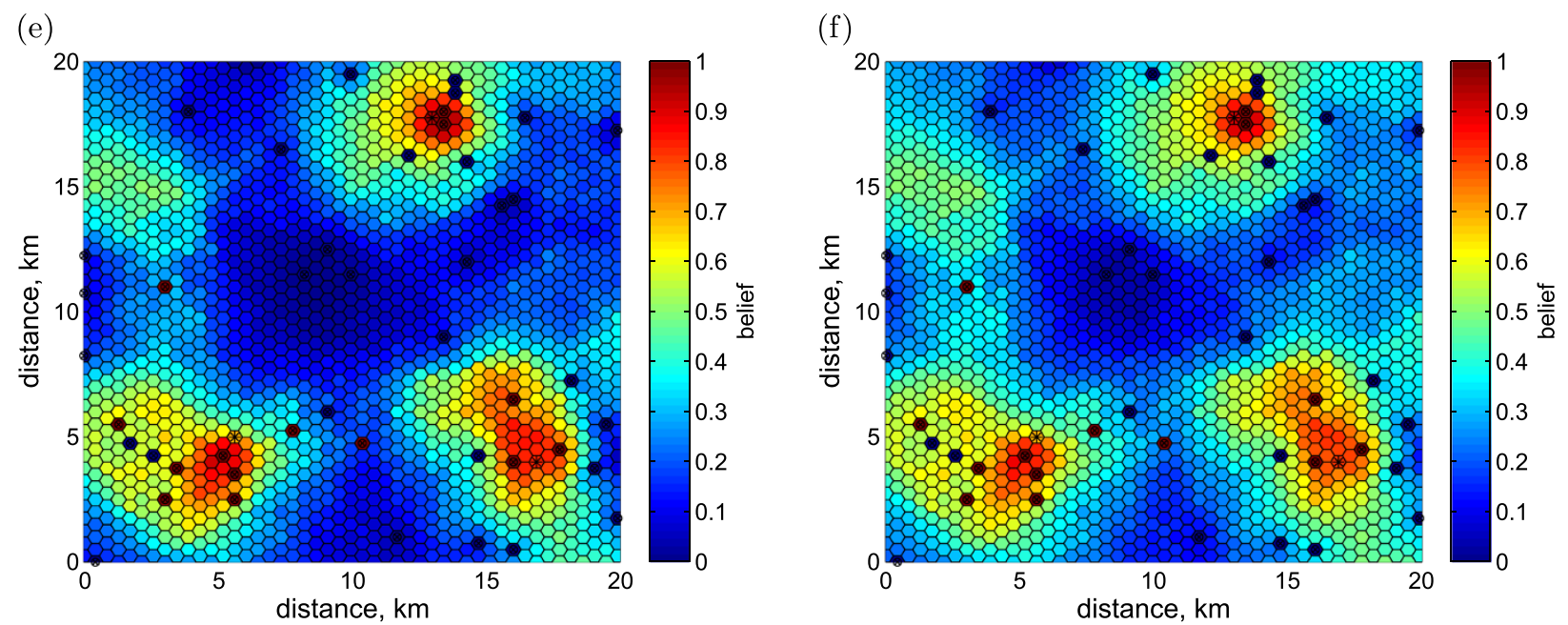

Figure 6 Results of Dempster-Shafer analysis for shadowing and sensing inaccuracies. Results of Dempster-Shafer analysis for example including shadowing with standard deviation $10 \mathrm{~dB}$, sensor accuracy and location errors. (a) True power levels (dBm). (b) True boundaries for $P_{\text {thresh }}=-60 \mathrm{dBm}$. (c) Belief that power exceeds $P_{\text {thresh }}$ for $(\Sigma+\Phi)=0$. (d) Belief that power exceeds $P_{\text {thresh }}$ for $(\Sigma+\Phi)=10$. (e) Belief that power exceeds $P_{\text {thresh }}$ for $(\Sigma+\Phi)=20$. (f) Belief that power exceeds $P_{\text {thresh }}$ for $(\Sigma+\Phi)=30$. 
computed as described in the Appendix using the spatial constant $\delta=1,000 \mathrm{~m}$. The standard deviation of the shadowing loss is $10 \mathrm{~dB}$. The regions above and below the threshold $P_{\text {thresh }}=-60 \mathrm{dBm}$ are shown in Figure 6b.

The belief functions for different values of $(\Sigma+\Phi)$ are shown in Figure $6 \mathrm{c}, \mathrm{d}, \mathrm{e}, \mathrm{f}$. The maximum combined absolute pathloss uncertainties due to sensor errors and shadowing observed in this simulation are approximately $8 \mathrm{~dB}$. When no account is taken of the pathloss variations due to shadowing and sensor errors, Figure 6c, the regions around the emitters are clearly identified, but there are cells which are seen from Figure $6 \mathrm{~b}$ to be above $P_{\text {thresh }}$ which are identified with strong belief as below the threshold. As $(\Sigma+\Phi)$ is increased, the belief functions become less polarised: Figure $6 \mathrm{f}$ shows that for large $(\Sigma+\Phi)$, the belief function indicates less confidence in the boundaries around the emitters and the spectrum manager is presented with a less definitive view of the spectrum occupancy. This appears to be appropriate, as the true boundaries are less well defined compared to the flat-earth case of Section 4.

\section{Parameter selection}

The reliability measure, $r$, was introduced in (11a) to weight the evidence from different sources. This is a heuristic measure, based on the recognition that sensors further away should have less influence on the local belief function than those closer. In (12) the parameter $\beta$ was used as an exponent in the computation of $r$. Figure 7 shows the dependence of the belief function distributions on $\beta$, with the results accumulated over ten realisations, each with between 1 and 4 emitters, with transmit powers between 15 and $25 \mathrm{~W}$. The shadowing field was randomly generated for each realisation, as described in the

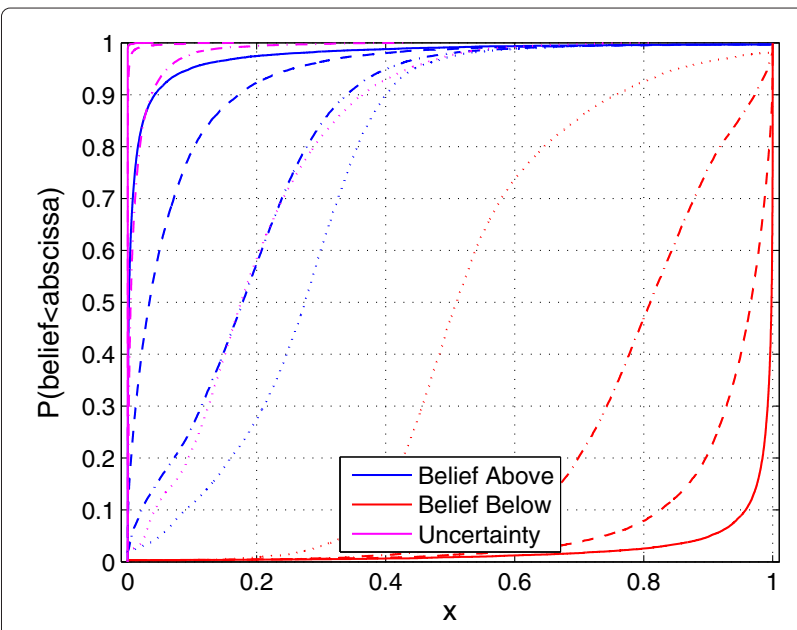

Figure $7 \mathrm{CDF}$ s of belief functions. $\beta=0.1$ (solid lines), $\beta=0.25$ (dashed lines), $\beta=0.5$ (dash-dotted lines) and $\beta=1$ (dotted lines) for $N=50$.
Appendix, with shadow loss standard deviation of $4 \mathrm{~dB}$, pathloss exponent $\alpha=3$ and sensor errors as in the previous simulations. The values used in computing the evidence were $\hat{\alpha}=2.8,(\Sigma+\Phi)=20$.

When $\beta$ is very small $(0.1)$, distant sensors have very little impact on the belief functions, which are therefore computed with very little evidence. As little uncertainty is accounted for, the belief functions are highly polarised (bel $\{A\}$ and bel $\{B\}$ near zero or unity) and consequently fail to alert to some regions of interest. At the other extreme, when $\beta$ is too high (1.0), the distant sensors have too much influence, introducing contradictory evidence and resulting in more neutral belief functions $(\operatorname{bel}\{A\} \approx$ bel $\{B\}$ ), which does not help the spectrum manager to discriminate regions of interest. The value of $\beta=0.5$ was selected as a balance between these two extremes and was used in the results throughout this paper, but it should be noted that there is not a 'correct' selection of this parameter, and any attempt to optimise it would be highly dependent on the specific assumptions in the scenario.

The parameter $D_{\mathrm{s}}$ is related to $\beta$ through (12). $D_{\mathrm{s}}$ is the maximum distance of sensors whose evidence will be considered. It should be selected to be large enough that the reliability at the distance $D_{\mathrm{s}}$ from the cell of interest will be small. For the idealised example here, $D_{\mathrm{s}}$ was selected to be $10 \mathrm{~km}$, at which distance the reliability $r \approx 0.22$. Extensive simulations showed there was no noticeable advantage in including sensors further away, but excluding them reduced the total computation required. In the following section, a larger $D_{\mathrm{s}}$ is used to counter the lower density of sensors.

The computational complexity for each cell is dominated by the sensor combining, which is dependent on the number of sensors within range. This can be controlled by using only the nearest, hence most reliable, $N$ sensors, with $N$ fixed.

\section{Emulated propagation environment}

The results presented thus far have used standard models to represent the ground truth of the propagation conditions. In real environments, the propagation characteristics are far more complex and are not uniform over the area.

To test this belief-based approach to spectrum occupancy mapping for more realistic conditions, a more sophisticated propagation environment has been considered. As collecting detailed spectral occupancy measurements over a large area is not feasible, the propagation prediction program CRC-COVLAB ${ }^{\mathrm{mm}}[30]$ was used to generate the ground truth. CRC-COVLAB takes into account the terrain and ground cover in predicting signal power over a wide geographic area. 
The area selected was a $60 \times 45 \mathrm{~km}$ rural area centred about $35 \mathrm{~km}$ west of downtown Ottawa, Canada, as shown in Figure 8. Particular features in the map include the Ottawa River, crossing from west to east approximately $20 \mathrm{~km}$ from the top edge of the area and the Eardley Escarpment, seen in the northeast of the map, where there is a steep increase in elevation of up to $270 \mathrm{~m}$. This area was divided into 3,060 hexagonal cells with centres separated by $d_{\text {hex }}=1,000 \mathrm{~m}$. Three emitters with isotropic antennas mounted at $3 \mathrm{~m}$ above the ground were located as shown in Figure 8, each operating at $100 \mathrm{~W}$ effective isotropic radiated power at a carrier frequency of $300 \mathrm{MHz}$.

The power levels output by the prediction program are shown in Figure 9a, and the cells with power above $P_{\text {thresh }}=-80 \mathrm{dBm}$ are indicated in Figure $9 \mathrm{~b}$. The $N=$ 100 sensors were randomly located in the area, ensuring only a single sensor per cell, which is positioned in the centre of the cell. As in Section 5, the sensors were assumed to be subject to measurement errors, which are uniformly distributed in $[-3,3] \mathrm{dB}$. The parameters used in the DS SOM process were as follows: $\hat{\alpha}=3.0, \beta=0.5$, $D_{\mathrm{s}}=12,000 \mathrm{~m}, P_{\mathrm{e}, \min }=10 \mathrm{~W}, P_{\mathrm{e}, \max }=150 \mathrm{~W}$.

Figure 9c,d shows the averaged belief functions bel $\{A\}$ for 100 sets of random sensor locations using $(\Sigma+\Phi)=$

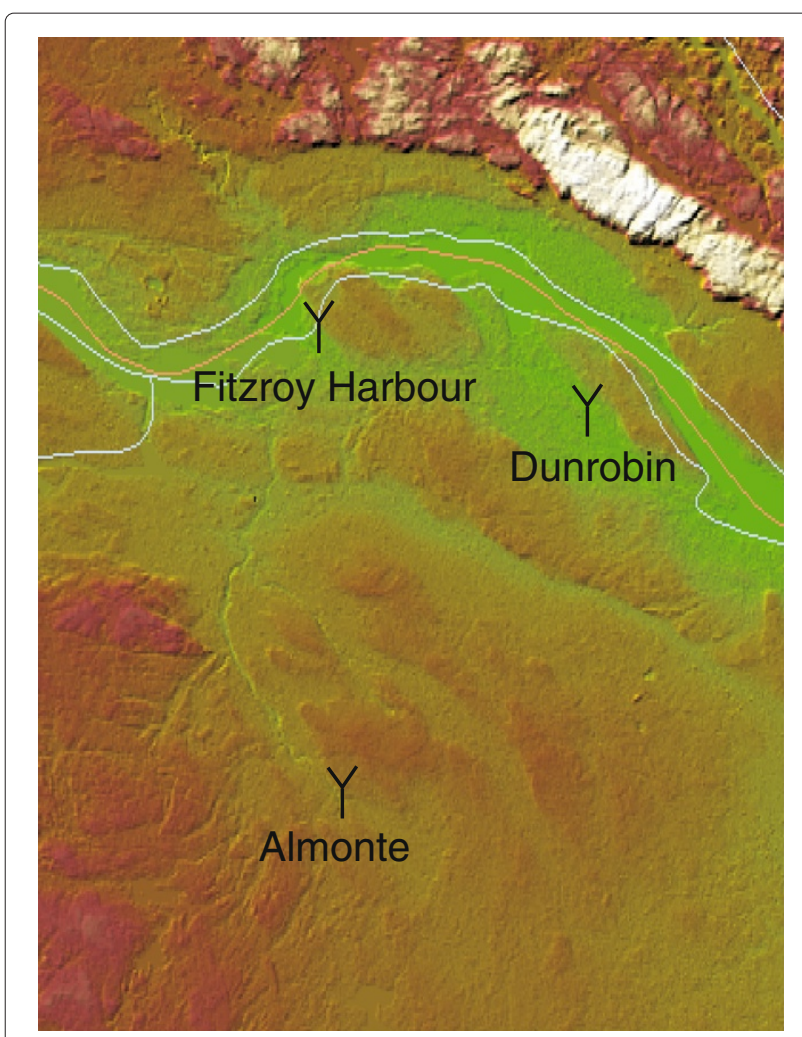

Figure 8 Terrain map of real propagation example.
40 and 20, respectively. With the larger value of $(\Sigma+\Phi)$, a larger number of cells is identified as having power levels possibly greater than $P_{\text {thresh }}$, while at the lower value, the belief function is more focussed on the transmission regions. This is expected from the results in the previous section. The maximum variance across the whole region is 0.053 in Figure 9c and 0.10 in Figure 9d, and the highest variance is observed where there is a lack of discrimination, i.e. where $\operatorname{bel}\{A\} \approx \operatorname{bel}\{B\}$. These averaged results do not show a clearly identifiable region corresponding to the Eardley Escarpment, and there is less confidence in the area around the Almonte transmitter, $(19,14) \mathrm{km}$, compared to the northern transmitters. Figure $9 \mathrm{~b}$ shows the effect of the terrain around the southwest region, which has a smaller range compared to the Dunrobin transmitter at $(31,36) \mathrm{km}$ and is therefore harder to identify with confidence and has average belief $\operatorname{bel}\{A\} \approx 0.6 \mathrm{com}$ pared to greater than 0.75 in the northern regions for $\Sigma+\Phi=40$.

Interestingly, this realistic scenario is more tolerant to variations in the estimated pathloss parameter, $\hat{\alpha}$, than the more idealistic models considered earlier. In particular, as $\hat{\alpha}$ is increased from 2.5 to 4.0, a larger percentage of the total area is identified as above $P_{\text {thresh, }}$ with a strong confidence. The additional areas are those with true power levels close to $-80 \mathrm{dBm}$, such as those in the region between the emitters. There is also an increased indication that power levels in the region around the Eardley Escarpment exceed the threshold.

For a comparison with power interpolation methods to generating a radio environment map, the modified Shepard's method (MSM) has been applied to the same emulated environment. This method was proposed in [31] for interpolation of scattered data and applied to the indoor radio environment map problem in [7]. MSM fits a spatial power function around each sensor, using weighted measurements from the sensors within a range $R_{q}$. For each cell without a sensor, the functions for each sensor within a distance $R_{w}$ are combined using a distance-dependent weighting. In this implementation, the parameters used were $R_{q}=12 \mathrm{~km}$ and $R_{w}=$ $8 \mathrm{~km}$, which correspond to average numbers of nodes $N_{q}=11$ and $N_{w}=5$ for this area and $N=100$ sensors. The weight function for cell $c_{i}$ and the sensor in cell $c_{j}$ was

$$
w_{i, j}=\left(\frac{R-d_{i, j}}{R d_{i, j}}\right)^{d_{\exp }} \quad d_{i, j} \leq R
$$

for both the spatial function computation $\left(R=R_{q}\right)$ and the power estimation $\left(R=R_{w}\right)$, where $d_{i, j}$ is the Euclidian distance from cell $c_{i}$ to cell $c_{j}$. As in [7], linear spatial functions were used and $d_{\exp }=1$ in (17). 

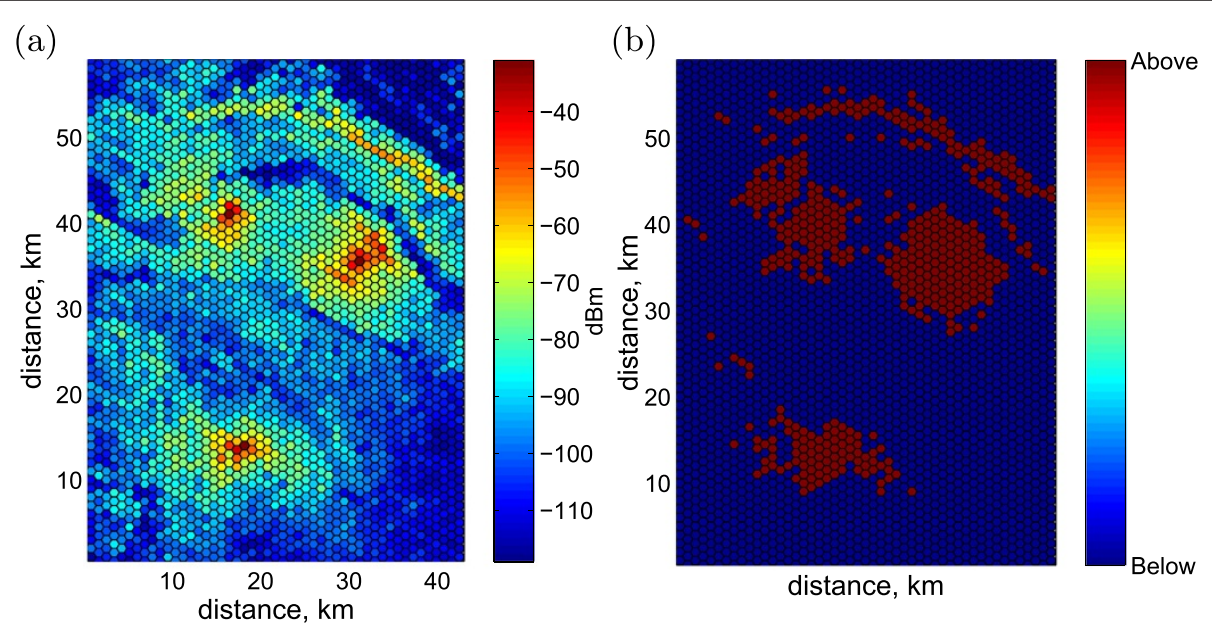

(c)

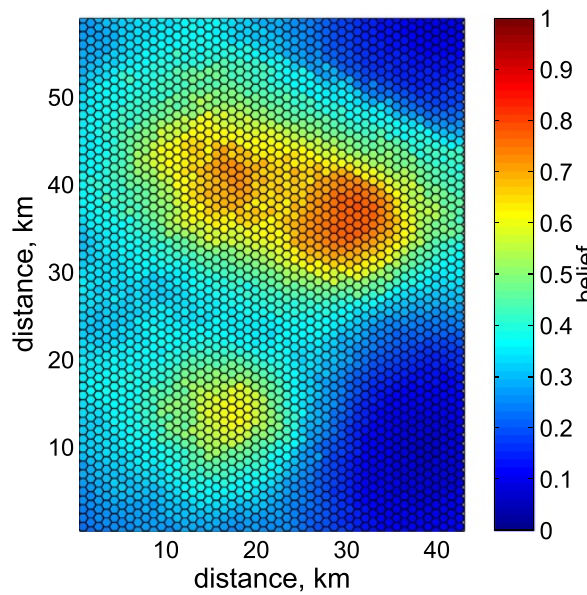

(d)

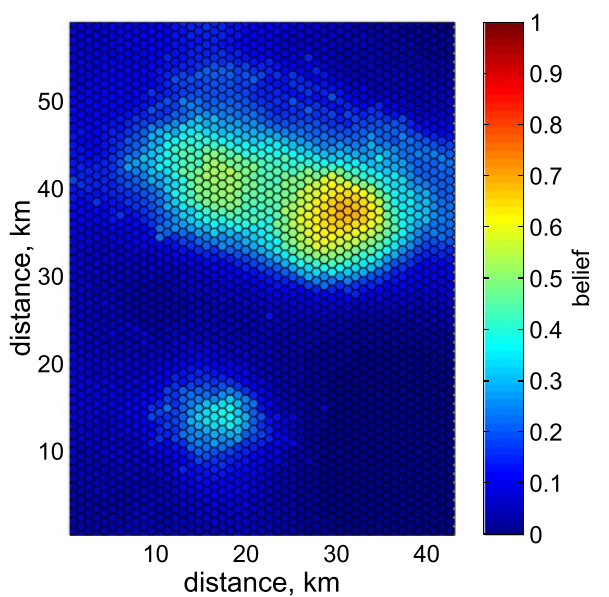

Figure 9 Results of Dempster-Shafer analysis for emulated propagation environment. Results of Dempster-Shafer analysis averaged over 100 random sensor locations, for real propagation example with $N=100$. (a) True spectrum map. (b) True boundaries. (c) Belief that power exceeds $P_{\text {thresh }}(\Sigma+\Phi)=40 .(\mathbf{d})$ Belief that power exceeds $P_{\text {thresh, }}(\Sigma+\Phi)=20$

The resulting radio environment map showing estimated power levels is shown in Figure 10a, averaged over 100 sets of random sensor locations. The regions around the three transmitters are identifiable, and the effect of the Eardley Escarpment is also visible. The variance of the estimates is shown in Figure 10b. The variance is highest around the perimeter where there are insufficient sensors to establish good spatial functions: the maximum variance in these regions exceeds 1,800 , but the scale has been modified to show greater resolution in the central regions. In those central regions, the variance is highest where the actual power is high, i.e. near the transmitters.

One challenge observed with the MSM technique is that the fitting process fails when there are insufficient neighbouring sensors within range $R_{q}$ - at least two are required to successfully generate spatial functions for each sensor.
Further, when there are insufficient sensors within range $R_{w}$ of a cell for the power estimation stage, the estimation fails to generate any value. The results in Figure 10 are averaged only over valid estimates and exclude the failed cases. Figure 10c shows the proportion of simulations that identified the power in each cell as exceeding $P_{\text {thresh. }}$ The regions around the transmitters at Dunrobin and Almonte are reliably identified, and the Fitzroy Harbour transmitter is identified in over $80 \%$ of cases. The cells in the challenging Eardley Escarpment region have a success rate of 50 to $60 \%$.

The DS approach does not provide absolute values for the state of the estimated power in a cell but rather a confidence level that the power exceeds or falls below a given threshold. It is possible to specify a belief threshold $b$ and make a decision based on $\operatorname{bel}\{A\} \geq b$, as an 

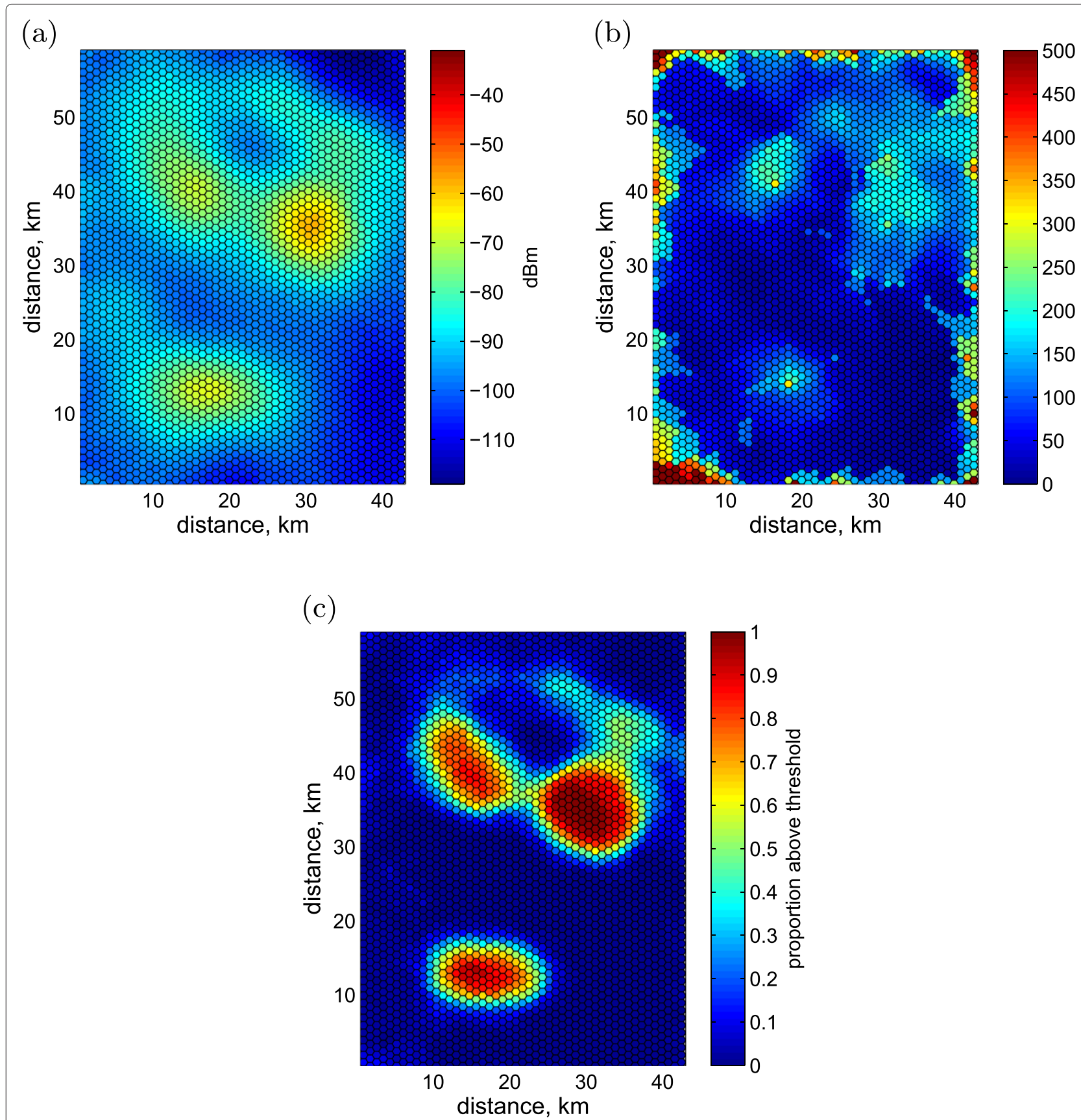

Figure 10 Comparison with power interpolation technique [7] for real propagation example, $\boldsymbol{N}=\mathbf{1 0 0}$. (a) Mean estimated power levels. (b) Variance of estimated power levels. (c) Rate of estimated power exceeding $P_{\text {thresh. }}$.

example, but it is stressed that this simplifies the richness of insight available from this technique. However, for the purposes of comparison, the incorrect 'detection' rate is used, i.e. the proportion of the cells for which the power is actually above $P_{\text {thresh }}$, but which are determined to be below and vice versa. This was computed for 100 random sets of sensor locations, for each method, using the parameters given above for MSM, and $\Sigma+$
$\Phi=40$ with $b=0.6$ for the proposed DS approach. The percentage of cells incorrectly identified is shown in Table 1.

The results above were obtained with randomly located sensors, which is most representative of a real mobile operating environment. If there were infrastructure available, the sensor locations could be selected and maintained on regular grid, as considered, for example, in 
Table 1 Percentage of cells incorrectly identified

\begin{tabular}{lcr}
\hline & Above (\%) & Below (\%) \\
\hline DS analysis & 16.2 & 8.4 \\
MSM & 42 & 9 \\
\hline
\end{tabular}

$[6,17]$. Different strategies for locating the sensors, taking into account both environment sensing and connectivity, were considered in [32]. Stehman compared random vs. regular sampling locations for other types of problems, for example in [33], and concluded that systematic, or regular, locations tend to be more accurate. Figure 11 shows the
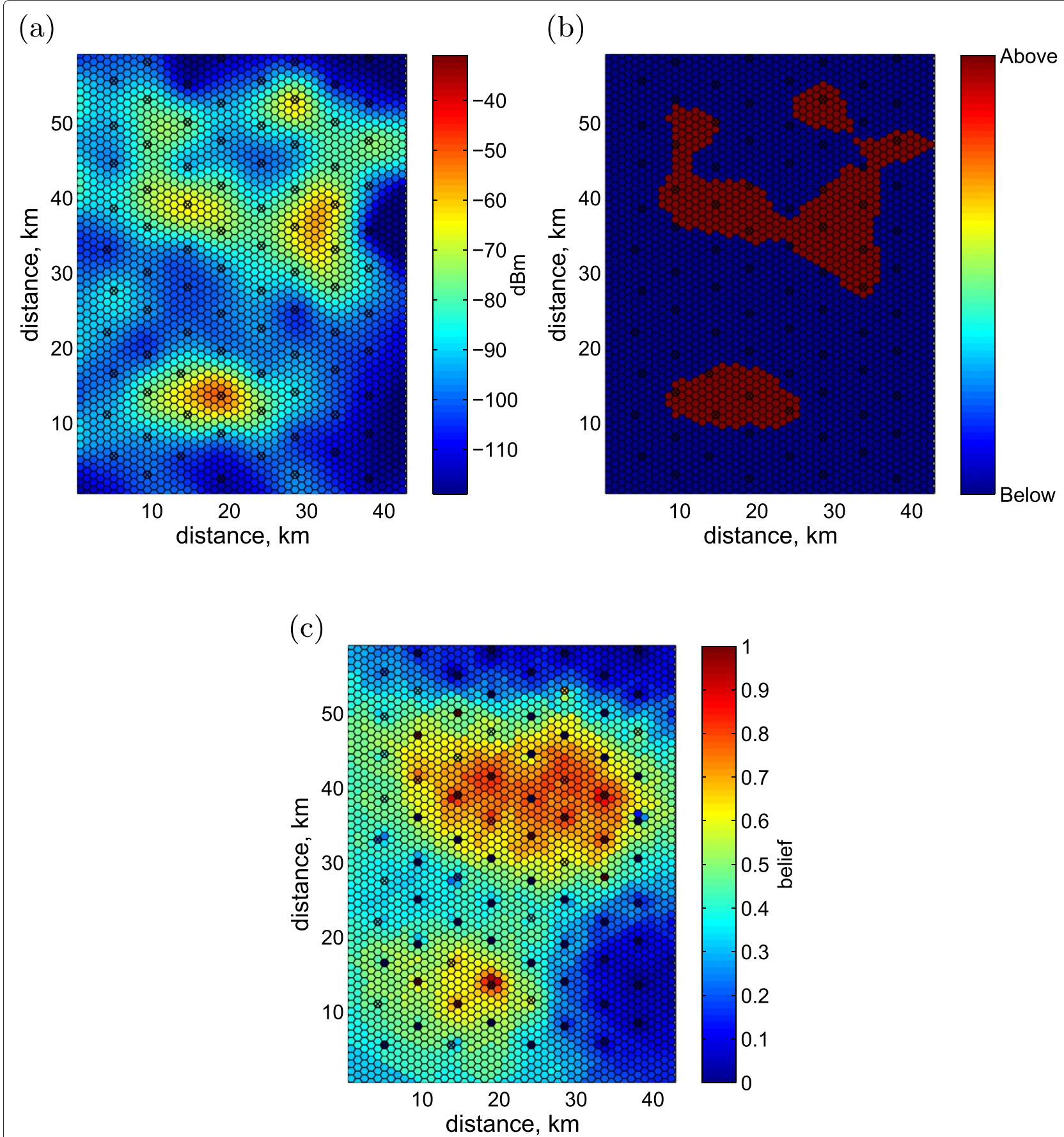

Figure 11 Sensors uniformly located on a lattice. (a) MSM estimated power. (b) Regions where MSM estimated power exceeds $P_{\text {thresh. }}$ (c) Belief that power exceeds $P_{\text {thresh }}, \Sigma+\Phi=40$. 
emulated performance of the MSM and DS-based analysis, where the 100 sensors are located on a hexagonal lattice. It is seen that, as expected, the estimation characteristics of both are superior to the averaged random case.

\section{Conclusions}

This work has investigated the application of a beliefbased approach, the Dempster-Shafer theory, to the problem of computing spectrum occupancy maps for heterogeneous mobile radio environments. In contrast to previous work, this approach considers the confidence, or belief, in the two hypotheses: the power is above the specified threshold, or the power is below. This also allows for a concept of uncertainty, which itself can be useful, as it indicates the sufficiency of evidence available to support either hypothesis.

The information provided to the spectrum manager is only as good as the data provided by the sensors, which means that when the sensors are particularly sparse in a given area, the resulting SOM can provide only hints rather than clear indications of spectrum occupancy. The DS method proposed here has this capability: by showing a high level of uncertainty, the spectrum manager knows there is insufficient coverage in this area and can make decisions accordingly. Further, it is clear where additional sensors should be deployed, if possible.

This work has also illustrated the challenges of generating spectrum occupancy maps: the propagation conditions in real environments do not follow the statistical models typically used in channel simulations, and suitable parameters are not known. Approaches to generating a SOM that rely on the accuracy of standard channel models will therefore fail in many real environments.

The Dempster-Shafer approach uses only a simple exponential pathloss model and does not rely on accurate parameterisation. The selection of the propagation parameters such as pathloss exponent and combined error effects $(\Sigma+\Phi)$ has an impact on the resulting belief functions, but useful information is provided to support the spectrum management function over a wide range of values. An effective implementation would be to enable these parameters to be varied to facilitate the interpretation of the analysis for complex environments. Furthermore, over large regions, the propagation characteristics can vary considerably, and the DS method allows different parameters in different regions.

For mobile environments, updating the SOM should focus on the areas of most significance, for example, those cells which have near-neutral belief functions resulting from conflicting evidence. In the DS approach proposed here, the cells are processed independently, which facilitates efficient updating of the map as sensors and emitters to move by computing belief functions for only a subset of cells at any time.

It is clear that the accuracy and resolution of a spectrum occupancy map are limited by the number and placement of sensors. A regular grid of sensors is likely to provide the most information, but this requires sensors to be pre-deployed, which may not be feasible in practice. Ultra-low power devices may also be missed unless the sensors are densely located. The spectrum management function using the SOM must therefore have sufficient intelligence and context to interpret the information it provides and make appropriate decisions.

A comparison with the modified Shepard's method of power interpolation has shown that the proposed DS approach is relatively robust to variations in sensor location. When there is insufficient sensor coverage, the uncertainty value becomes non-negligible, alerting the spectrum manager to that area.

The spectrum management function is necessarily subjective, as the risks of interference must be balanced with the requirement to provide bandwidth to priority users. One of the advantages of the Dempster-Shafer method is that it enables a sophisticated interpretation of the spectrum occupancy, which supports context-specific decision-making.

\section{Appendix}

\section{Shadow fading model}

Most shadow fading models address only a single link; where there is more than one link, they are assumed to share a common terminal, e.g. [34]. In the case of the spectrum occupancy map problem, there are many links of interest but they do not all share a single terminal. This is the problem addressed in [35], however in that model the link fading is purely statistical which means the relative positions of the link terminals are not specifically taken into account.

Herein, a spatial shadow fading correlation method is proposed that is based on the fundamentals of the approach in [35], but maintains the spatial relationship of terminals. The approach in [35] was based on an underlying spatial loss field. This models the loss experienced on a link that passes through a given point or region on the field such that the normalised shadowing loss on the link between $\mathbf{x}_{i}$ and $\mathbf{x}_{j}$ is given by

$$
S L_{i, j}=\frac{1}{\left\|\mathbf{x}_{i}-\mathbf{x}_{j}\right\|^{\frac{1}{2}}} \int_{\mathbf{x}_{i}}^{\mathbf{x}_{j}} p(\mathbf{x}) d \mathbf{x} .
$$


As in [35], the spatial loss field $p(\mathbf{x})$ is modelled as a widesense stationary Gaussian random field with zero mean. The spatial correlation is modelled as an exponential decay, i.e.

$$
E\left\{p\left(\mathbf{x}_{i}\right) p\left(\mathbf{x}_{j}\right)\right\}=\frac{\sigma^{2}}{\delta} \exp \left(-\frac{\left\|\mathbf{x}_{i}-\mathbf{x}_{j}\right\|}{\delta}\right)
$$

where $\delta$ is a spatial constant and $\sigma$ is the standard deviation of the shadowing loss.

Each spatial field realisation is generated by generating the covariance matrix $\mathbf{C}_{p}$ for the locations of interest (centres of hex cells, in this case) using (19). The spatial losses are computed as

$$
\tilde{p}=\mathbf{C}_{p}^{\frac{1}{2}} \mathbf{g}
$$

where $\mathbf{g}$ is a vector of zero-mean, unit-variance Gaussian variables. The square root of $\mathbf{C}_{p}$ is found as the lower triangular matrix $\mathbf{L}$ in the Cholesky decomposition $\mathbf{C}_{p}=$ $\mathbf{L L}^{T}$.

In the simulation, the shadowing loss between two points $\mathbf{x}_{i}$ and $\mathbf{x}_{j}$ is estimated using

$$
\widetilde{S L}_{i, j}=\frac{1}{\left\|\mathbf{x}_{i}-\mathbf{x}_{j}\right\|^{\frac{1}{2}}} \sum_{\ell \in L} p\left(\mathbf{x}_{\ell}\right) \Delta
$$

where $L$ is the set of points spaced by $\Delta$ along the straight line between $\mathbf{x}_{i}$ and $\mathbf{x}_{j}$. As shown in [35], for points separated by more than the spatial correlation distance, i.e. $\left\|\mathbf{x}_{i}-\mathbf{x}_{j}\right\| \gg \delta$, the variance of the shadowing loss is $\operatorname{var}\left\{\widetilde{S L}_{i, j}\right\} \approx \sigma^{2}$.

\section{Competing interest}

The author declares that she has no competing interest.

\section{Acknowledgements}

This work was supported by Defence R\&D Canada.

Received: 19 October 2012 Accepted: 25 March 2013

Published: 24 April 2013

\section{References}

1. BA Fette, Cognitive Radio Technology, 2nd edn. (Academic Press, Burlington, 2009)

2. W Krenik, A Batra, in Proceedings of 42nd Design Automation Conference (DAC). Cognitive radio techniques for wide area networks (Anaheim, CA, USA, 2005), pp. 409-412

3. A Alaya-Feki, SB Jemaa, B Sayrac, P Houze, E Moulines, in Proceedings of 19th IEEE International Symposium on Personal, Indoor and Mobile Radio Communications (PIMRC). Informed spectrum usage in cognitive radio networks: interference cartography (Cannes, France, September 2008), pp. $15-18$

4. FARAMIR Project 2010, Flexible and spectrum aware radio access through measurements and modelling in cognitive radio systems. http://www.ict-faramir.eu/ Accessed 15 November 2011

5. E Meshkova, J Ansari, D Denkovski, J Riihijärvi, J Nasreddine, M Pavloski, L Gavrilovska, P Mahonen, in Proceedings of IEEE DySPAN 2011. Experimental spectrum sensor testbed for constructing indoor radio environmental maps (Aachen Germany, 3 -6 May 2011), pp. 603-607

6. C Phillips, M Ton, D Sicker, D Grunwald, in Proceedings of IEEE DySPAN 2012. Practical radio environment mapping with geostatistics (Bellevue WA, USA, 16 -19 October 2012), pp. 422-433
7. D Denkovski, V Atanasovski, L Gavrilovska, J Riihijarvi, P Mahonen, in Proceedings of International Conference on Cognitive Radio Oriented Wireless Networks (CROWNCOM). Reliability of a radio environment map: case of spatial interpolation techniques (Stockholm Sweden, 18 -20 June 2012), pp. 248-253

8. G Mateos, JA Bazerque, GB Giannakis, in Conference Record of the Forty-Third Asilomar Conference on Signals, Systems and Computers. Spline-based spectrum cartography for cognitive radios (Pacific Grove CA, USA, 1 -4 November 2009), pp. 1025-1029

9. JA Bazerque, GB Giannakis, Distributed spectrum sensing for cognitive radio networks by exploiting sparsity. IEEE Trans. Signal Process. 58(3), 1847-1862 (2010)

10. $Y$ Ran, $X L i, L L u, Z$ Bai, in Proceedings of International Symposium on Spatial Accuracy Assessment in Natural Resources and Environmental Sciences. Land cover classification information decision making fusion based on Dempster-Shafer theory: results and uncertainty (Shanghai, China, 25 -27 June 2008), pp. 240-247

11. SB Chaabane, M Sayadi, F Fnaiech, E Brassart, Dempster-Shafer evidence theory for image segmentation: application in cells images. Int. J. Inform. Commun. Eng. 5(2), 126-132 (2009)

12. H Guo, W Shi, Y Deng, Evaluating sensor reliability in classification problems based on evidence theory. IEEE Trans. Syst., Man Cybern. B. 36(5), 970-981 (2006)

13. P Qihang, Z Kun, W Jun, L Shaogian, in Proceedings of 17th IEEE International Symposium on Personal Indoor and Mobile Radio Communications. A distributed spectrum sensing scheme based on credibility and evidence theory in cognitive radio context (Helsinki, Finland, $11-14$ September 2006)

14. X Zheng, J Wang, Q Wu, J Chen, in Proceedings of IEEE Singapore International Conference on Communication Systems. Cooperative spectrum sensing algorithm based on Dempster-Shafer theory (Guangzhou, China, 19 -21 November 2008), pp. 218-221

15. N Nguyen-Thanh, I Koo, An enhanced cooperative spectrum sensing scheme based on evidence theory and reliability source evaluation in cognitive radio context. IEEE Commun. Lett. 13(7), 492-494 (2009)

16. J Li, J Liu, K Long, in Proceedings of IEEE Global Telecommunications Conference. Reliable cooperative spectrum sensing algorithm based on Dempster-Shafer theory (Miami, FL, USA, 6-10 December 2010)

17. M Zhang, S Zhang, I Cao, in Proceedings of IEEE International Conference of Internet Computing in Science and Engineering. Fusing received signal strength from multiple access points for WLAN user location estimation (Harbin, China, $29-28$ January 2008), pp. 173-180

18. G Shafer, A Mathematical Theory of Evidence. (Princeton University Press, Princeton, 1976)

19. AP Dempster, A generalization of Bayesian inference. J. Roy. Stat. Soc. Ser. B. 30(2), 205-247 (1968)

20. J Pearl, Reasoning with belief functions: an analysis of compatibility. Int. J. of Approx. Reasoning. 4, 363-389 (1990)

21. JY Halpern, R Fagin, Two views of belief: belief as generalized probability and belief as evidence. Al. 54, 275-317 (1992)

22. G Shafer, Perspectives on theory and practice of belief. Int. J. Approx. Reasoning. 4, 323-362 (1990)

23. AP Dempster, The Dempster-Shafer calculus for statisticians. Int. J. Approx. Reasoning. 48, 365-377 (2008)

24. P Smets, The combination of evidence in the transferable belief model. IEEE Trans. Pattern Anal. Mach. Intell. 12(5), 447-458 (1990)

25. JC Hoffman, RR Murphy, in SPIE Proceedings on Neural and Stochastic Methods in Image and Signal Processing II, vol. 2032. Comparison of Bayesian and Dempster-Shafer theory for sensing: a practitioner's approach, (1993), pp. 266-279

26. M Beynon, B Curry, P Morgan, The Dempster-Shafer theory of evidence: an alternative approach to multicriteria decision modelling. Omega. 28(1), 37-50 (2000)

27. D Koks, S Challa, An introduction to Bayesian and Dempster-Shafer data fusion. Technical report DSTO-TR-1436, Defence Science and Technology Organisation, Edinburgh, Australia (2005)

28. LA Zadeh, A mathematical theory of evidence (book review). Al Mag. 5(3), 81-83 (1984)

29. AF Molisch, Wireless Communications. (Wiley, Chichester, 2005) 
30. CRC-COVLAB coverage and interference analysis software. www. slideshare.net/dcamire/crccovlab-coverage-prediction-softwareseminar-at-industry-canada Accessed 27 May 2013

31. R Franke, $G$ Nielson, Smooth interpolation of large sets of scattered data. Int. J. Numer. Methods Engin. 15, 1691-1704 (1980)

32. ES Biagioni, G Sasaki, in Proceedings of Hawaii International Conference on System Sciences. Wireless sensor placement for reliable and efficient data collection (Big Island Hawaii, USA, 6 -9 January 2003)

33. SV Stehman, Comparison of systematic and random sampling for estimating the accuracy of maps generated from remotely sensed data. Photogrammetric Eng Remote Sensing. 82(9), 1343-1350 (1992)

34. F Fabbri, R Verdone, in IEEE Vehicular Technology Conference. The impact of correlated channel fluctuations on the connectivity of wireless ad-hoc networks (Barcelona, Spain, $26-29$ April 2009)

35. P Agrawal, N Patwari, Correlated link shadow fading in multi-hop wireless networks. IEEE Trans. Wirel. Commun. 8(8), 2024-4036 (2009) mapping. EURASIP Journal on Wireless Communications and Networking 2013 2013:112.

\section{Submit your manuscript to a SpringerOpen ${ }^{\circ}$ journal and benefit from:}

- Convenient online submission

- Rigorous peer review

- Immediate publication on acceptance

- Open access: articles freely available online

- High visibility within the field

- Retaining the copyright to your article 